\title{
INOVAÇÃO SUSTENTÁVEL: UMA PERSPECTIVA COMPARADA DA LITERATURA INTERNACIONAL E NACIONAL
}

\section{Vanessa Cuzziol Pinsky}

Doutoranda em administração na Faculdade de Economia, Administração e Contabilidade da Universidade de São Paulo - FEA/USP

vanessa.pinsky@usp (Brasil)

\section{Sérgio Luiz do Amaral Moretti}

Doutorado em Ciências Sociais pela Pontifícia Universidade Católica de São Paulo, Brasil - PUC Professor da Universidade Anhembi Morumbi - UAM

sergiomoretti@uol.com.br (Brasil)

\section{Isak Kruglianskas}

Doutorado em Administração pela Universidade de São Paulo - USP

Professor da Universidade de São Paulo - USP

ikruglia@gmail.com (Brasil)

\section{Guilherme Ary Plonski}

Doutor em Engenharia de Produção pela Escola Politécnica da Universidade de São Paulo - USP Professor da Faculdade de Economia, Administração e Contabilidade - FEA/USP plonski.usp@gmail.com (Brasil)

\section{RESUMO}

A inovação é um dos principais fatores que influencia positivamente a competitividade e o desenvolvimento econômico. A inovação sustentável apresenta-se como uma alternativa para a construção de uma nova economia de baixo carbono, visando ao desenvolvimento sustentável e à viabilização de iniciativas com foco na mitigação e adaptação aos impactos ambientais, especialmente aqueles relacionados com às mudanças climáticas. $\mathrm{O}$ objetivo deste estudo foi realizar uma pesquisa bibliográfica sobre o conceito de inovação sustentável. Foi utilizado o método bibliométrico, com o intuito de facilitar o levantamento e seleção dos artigos científicos relevantes. Foi analisada quantitativamente a produção acadêmica internacional no período entre 2008 e abril de 2013 , e a produção brasileira até junho de 2014. Os resultados principais mostraram que há carência de padronização das terminologias e abrangência conceitual sobre inovação sustentável, a produção brasileira ainda é incipiente e o maior volume de produção sobre o tema está concentrado nas publicações da Holanda e dos Estados Unidos. Os principais aspectos deste estudo são: contribuir com uma sistematização de maior qualidade nas pesquisas bibliográficas, destacar a importância de trabalhar com autores e periódicos que possuam relevância para os seus respectivos campos de estudo e adotar trabalhos que estão na fronteira do conhecimento. Em particular, destacaram-se os principais autores e periódicos no tema da inovação sustentável. O resultado do estudo possibilita estabelecer camadas de aprofundamento das análises de citações, permitindo encontrar relações entre as linhas mestras nas quais o campo se apoia, além de relacionar a produtividade dos autores por meio das coautorias.

Palavras-chave: Inovação Sustentável; Eco inovação; Sustentabilidade; Inovação Verde; Bibliometria. 


\section{INTRODUÇÃO}

A inovação sustentável demanda a integração de competências ao longo da cadeia produtiva da empresa, na qual a regulação ambiental incentiva a utilização de novos materiais e tecnologias, diferentes funcionalidades e usos e descartes para os produtos (Gonçalves-Dias, Guimarães \& Santos, 2012).

Segundo Schaltegger e Wagner (2011) há três razões fundamentais para a inovação sustentável quando relacionada ao benefício privado de redução de custos e o benefício social que é gerado, a saber: 1) o benefício privado é maior do que o impacto negativo gerado pela inovação; 2) uma inovação tem um impacto social positivo maior do que o benefício privado; e, 3) os benefícios privado e social se equiparam. As razões expostas pelos autores são, por seu lado, os vetores que determinam a análise estratégica pelas empresas.

Na cúpula Rio+20 em 2012, sob a liderança da ONU, foram criados os Objetivos de Desenvolvimento Sustentável (ODS), um conjunto de metas, em processo de finalização que deverá ser assumido por todos os países-membros da ONU após 2015, quando expira o prazo para o cumprimento dos Objetivos do Milênio (ODM). As novas metas são baseadas em quatro dimensões que afetam particularmente as empresas: desenvolvimento econômico (incluindo o fim da pobreza extrema), inclusão social, sustentabilidade ambiental e boa governança, considerando a paz e a segurança. Diante desse contexto, é esperado pela sociedade civil que os ODS norteiem as políticas públicas e privadas das nações nos próximos anos, sendo que as inovações orientadas para a sustentabilidade vão ao encontro das expectativas e metas estabelecidas por meio dos 17 ODS. Em especial ao nono objetivo que é centrado na construção de uma infraestrutura resiliente, no fomento de processos produtivos sustentáveis e inclusivos por meio da inovação (UN, 2014).

Do ponto de vista da inovação sustentável, na vertente da sustentabilidade ambiental, o aumento da temperatura, o esgotamento dos limites de utilização de alguns sistemas terrestres, a taxa de perda da biodiversidade e a interferência humana no ciclo do nitrogênio podem trazer consequências irreversíveis e catastróficas para muitas partes do planeta, afetando principalmente regiões mais pobres e vulneráveis (IPCC, 2007; Rockström et al., 2009).

Por seu lado, a vertente da sustentabilidade social, apresenta um cenário complexo diante de um contingente populacional estimado em nove bilhões de pessoas até 2050 (WBCSD, 2010), que gerará aumento das expectativas e de atritos com relação ao fornecimento de energia, segurança alimentar, saneamento, moradia, disponibilidade de recursos naturais, degradação dos ecossistemas e

Revista de Administração e Inovação, São Paulo, v. 12, n.3 p. 226-250, jul./set. 2015. 
crescente desigualdade econômica. Esse cenário demandará consideráveis mudanças em produtos, serviços, processos e modelos de gestão das empresas (Seebode, Jeanrenaud \& Bessant, 2012).

Complementarmente, o aumento da competitividade global, a crescente demanda por eficiência operacional com redução de custo e qualidade, as regulações socioambientais, a pressão de partes interessadas (stakeholders) e a rápida transformação tecnológica desafiam cada vez mais as empresas a inovar com foco em sustentabilidade. Se por um lado o presente cenário é desafiador e complexo para as empresas, por outro, a inovação sustentável pode se tornar uma alternativa para estratégias de diferenciação e oportunidades de negócios, por meio da redução de custo e risco e de alinhamento com as expectativas da sociedade (Porter \& Van der Linde, 1995a, 1995b; Elkington, 1998, 2001; May, Lustosa \& Vinha, 2003; Hart \& Milstein, 2004; Aligleri, Aligleri \& Kruglianskas, 2009). Essa perspectiva é o principal fator e orientação para que as empresas gradativamente adotem práticas de negócios mais alinhadas com o equilíbrio das dimensões econômica, social e ambiental.

O efeito sistêmico dessas demandas pressiona as empresas a inovar nos seus processos produtivos e de gestão, envolvendo cada vez mais a sua cadeia de suprimentos na busca por soluções sustentáveis (Kiron et al., 2013). A inovação apresenta-se como um dos principais fatores que influenciam positivamente a competitividade e o desenvolvimento econômico. Países que fomentam a inovação buscam melhoria na produtividade, tornando suas economias mais eficientes. Empresas inovadoras também apresentam um melhor desempenho (PINTEC, 2013).

Deve-se ressaltar que a sustentabilidade cria vantagem competitiva para as empresas, gera valor para acionistas, consumidores e meio ambiente. Pesquisa recente conduzida pelo MIT Sloan Management Review e The Boston Consulting Group (Kiron et al., 2013).investigou como as empresas consideram a sustentabilidade relacionada à necessidade e oportunidade, e suas capacidades de implementação de novos modelos de negócios com sucesso. Os resultados evidenciam que o número de empresas que reportam lucro centrado em iniciativas de sustentabilidade aumentou de $23 \%$ para 37\% de 2012 para 2013. Além disso, metade dos respondentes indicou mudança no modelo de negócio como resultado de oportunidades com foco em sustentabilidade. Nesse sentido, a inovação torna-se crítica para o lucro centrado em novas práticas de sustentabilidade

Os desafios do desenvolvimento sustentável no contexto corporativo, considerando o potencial transformador e de contribuição das empresas diante das pressões socioambientais justificam este estudo. Por essa razão, o campo de conhecimento da administração, com foco na temática sustentabilidade e inovação, tem se tornado relevante e sua produção científica apresenta aumento significativo.

Revista de Administração e Inovação, São Paulo, v. 12, n.3 p. 226-250, jul./set. 2015. 
O objetivo deste estudo foi pesquisar o conceito de inovação sustentável e, também, apresentar um método de sistematização para o levantamento bibliográfico por meio de recursos bibliométricos. Espera-se que os resultados dessa aplicação prática auxiliem futuras pesquisas científicas, facilitando o levantamento de obras relevantes para a revisão de literatura.

O presente trabalho está estruturado da seguinte forma: revisão da literatura sobre inovação sustentável, procedimentos metodológicos, análise e discussão dos resultados e considerações finais.

\section{REVISÃO DA LITERATURA}

Para iniciar a revisão bibliográfica sobre inovação sustentável é necessário considerar a semântica e o próprio significado das palavras “inovação sustentável”. Embora polêmica, a definição de sustentabilidade mais difundida é baseada no equilíbrio das dimensões econômica, ambiental e social, o triple bottom line (Elkington, 1998; 2001). Já a inovação é impulsionada pela capacidade de detectar conexões, com o objetivo de identificar oportunidades de melhoria em produtos, serviços e processos, transformar ideias em realidade e aproveitá-las, não apenas por meio de novos mercados, mas considerando novas ofertas a mercados existentes (Tidd \& Bessant, 2009).

A Organização para a Cooperação e Desenvolvimento Econômico (OECD) define inovação como "a implementação de um produto (bem ou serviço) novo ou significativamente melhorado, ou um processo, ou um novo método de marketing, ou um novo método organizacional nas práticas de negócios, na organização do local de trabalho ou nas relações externas” (OSLO, 2004).

A revisão da literatura de inovação sustentável permitiu identificar uma falta de padronização das diferentes terminologias para definir o que vem a ser uma inovação sustentável. De maneira geral, a literatura pesquisada é centrada na inovação que possibilita a redução do impacto negativo sobre o meio ambiente, utilizando terminologias como 'verde', 'eco', 'ambiental' e 'sustentável' (Schiederig, Tietze \& Herstatt, 2012). Não foram identificados artigos abordando em profundidade a dimensão social em inovação sustentável. O enfoque central se concentra na relação das dimensões ambiental e econômica. Essa revisão foi centrada nas quatro palavras-chave mencionadas acima combinadas com a palavras inovação: verde, eco, ambiental e sustentável.

Tidd e Bessant (2009) argumentam que a abordagem convencional para inovação e sustentabilidade foca em instrumentos de comando e controle, por meio da regulação e punição pelo não cumprimento, tornando a mudança lenta e incremental nas empresas. Contudo, uma abordagem mais efetiva deveria focar na compreensão de como tecnologia, mercados e sociedade podem 
contribuir com inovações que tragam soluções para questões ambientais, incluindo produção mais limpa (redução do impacto ambiental no ciclo de vida), processos mais eficientes (redução de resíduos), tecnologias alternativas (redução de emissões), novos serviços (substituição ou redução do consumo de produtos) e inovação em sistemas (mensuração e monitoramento).

Um modelo de como a inovação pode contribuir com a sustentabilidade por meio da criação de novos produtos e serviços ou melhoria incremental, como proposto por Tidd e Bessant (2009), juntamente com a tipologia da inovação sustentável, é detalhado na Figura 1.

\section{Figura 1}

Tipologia de Inovações Sustentáveis

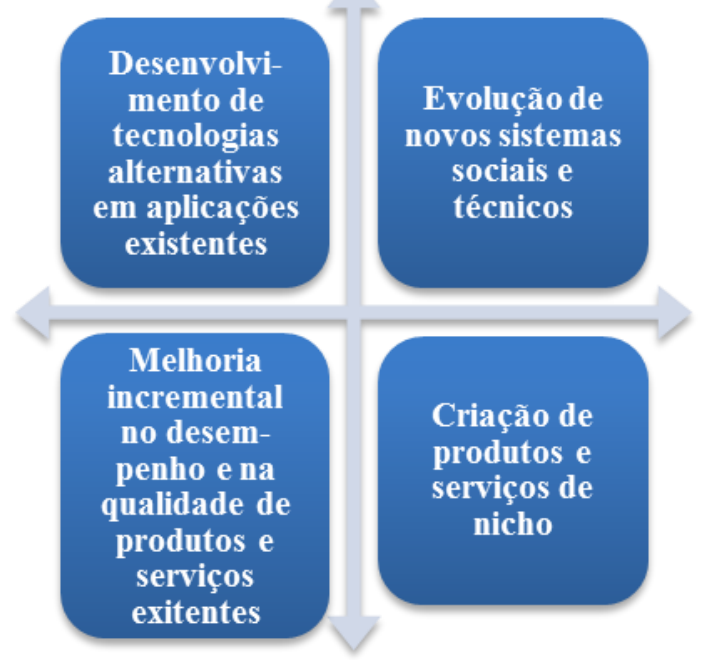

Fonte: Adaptado pelos autores de Tidd e Bessant (2009, p. 473)

Schot e Geels (2008) relacionam a gestão estratégica de nicho focada na utilização de novas tecnologias com seu alto potencial de contribuir com o desenvolvimento sustentável. A proposição é que a gestão estratégica de nicho pode facilitar a inovação sustentável por meio da modulação de nichos tecnológicos. Mas não se trata apenas de uma abordagem tecnológica. O desenvolvimento sustentável demanda mudanças tecnológicas e sociais concomitantemente. A indução de inovação sustentável, por meio da gestão estratégica de nichos deve ser feita de forma complementar aos incentivos de mercado e regulação.

Dentre as primeiras propostas para equalizar as necessidades empresariais e do meio ambiente e, portanto com foco no mercado, a Hipótese de Porter (HP) versa sobre a influência de uma regulação ambiental bem desenhada na competitividade empresarial. As empresas se beneficiam com a redução de custo e risco, por meio da inovação e cumprimento da regulação ambiental. A proposta é de mudança do controle da poluição para uma visão focada na produtividade dos recursos (Porter \& Van 
der Linde, 1995a). De maneira complementar Horbach (2007) postula que devido às externalidades decorrentes da degradação ambiental, a inovação ambiental é orientada predominantemente por regulação do que por mecanismos de mercado.

Mais recentemente Nidumolu, Prahalad e Rangaswami (2009) corroboram com a teoria central de que o cumprimento à regulação direciona a inovação como uma oportunidade de negócio, por meio da tecnologia, materiais e processos sustentáveis. No contraponto, Nill e Kemp (2009) argumentam que uma regulação ambiental não é suficiente para promover inovação sustentável. É latente a necessidade de promover mudanças radicais em tecnologia e nos sistemas de inovação visando ao desenvolvimento sustentável. A inovação incremental em setores consolidados não é suficiente para atender à demanda de metas de sustentabilidade ambiental, como a mitigação das mudanças climáticas.

De maneira semelhante é possível dialogar com Oltra e Jean (2009), que também questionam a HP. As inovações ambientais não podem ser consideradas como uma resposta simples e sistemática à pressão regulamentar, pois diversos fatores, tais como bases de conhecimento, oportunidades tecnológicas e demanda influenciam as respostas tecnológicas das empresas. Os autores definem inovação ambiental como processos novos ou modificados, práticas, sistemas e produtos que beneficiam o meio ambiente e contribuem com a sustentabilidade ambiental.

O desenvolvimento da inovação ambiental é muitas vezes direcionado por metas ambientais relacionadas à redução de custo, regulação, mecanismos de mercado ou instrumentos voluntários (Frondel et al., 2008). Neste sentido, a eco inovação é um conceito amplo, que inclui inovação no controle da poluição (tecnologias novas, melhores ou mais baratas), produtos verdes, tecnologias de produção limpa, tecnologias de energia e de transporte sustentáveis, redução de resíduos e técnicas de manejo. A inovação pode ser nova, radical ou incremental (Kemp \& Pontoglio, 2011).

Segundo Rennings (2000), a eco inovação aborda três tipos de mudanças para o desenvolvimento sustentável: inovação tecnológica, social e institucional. O autor traz à luz algumas peculiaridades da eco inovação, incluindo as externalidades dos problemas, os efeitos da regulação e a crescente importância da inovação no contexto social e institucional, sendo que a inovação para a sustentabilidade reduz o impacto ambiental.

Por seu lado, Chen, Lai e Wen (2006) consideram inovação verde em produtos e processos que envolvem inovação tecnológica com foco em eficiência energética, prevenção de poluição, reciclagem de resíduos, eco design e gestão ambiental corporativa. Já para Chen (2008) deve-se também levar em consideração que a competência principal verde de uma empresa está correlacionada positivamente com seu desempenho em inovação e imagem verde.

Revista de Administração e Inovação, São Paulo, v. 12, n.3 p. 226-250, jul./set. 2015. 
Para Kemp e Pearson (2007), autores do Measuring Eco-Innovation (MEI Report), uma das importantes referencias na temática, a base da definição de eco inovação é centrada no desempenho ambiental em detrimento das metas ambientais de uma empresa, pois o que interessa não são os objetivos e intenções corporativas, e sim os resultados ambientais positivos trazidos pela eco inovação. Os autores propõem uma classificação para os tipos de eco inovação, a saber:

a) Tecnologias ambientais, incluindo tecnologias para tratamento de água e resíduos, monitoramento ambiental, produção limpa, etc.;

b) Inovação organizacional para o meio ambiente, incluindo a introdução de novos métodos e sistema de gestão ambiental;

c) Inovação de produtos e serviços com uma proposta focada em benefícios ambientais;

d) Inovação de sistema verde (sustentável), incluindo sistemas alternativos de produção e consumo, e novos materiais.

Dessa maneira, todos os novos processos que apresentam mais eficiência na utilização dos recursos naturais são considerados eco inovação, sendo que a avaliação dos riscos e impactos ambientais, por meio da análise do ciclo de vida, contribui com esse processo. Seguindo esta linha, os autores oferecem uma definição de eco inovação:

[...] produção, assimilação e exploração de um produto, processo produtivo, serviço ou gestão ou método de negócio que é novo para uma organização (desenvolvimento ou adoção), e que resulta, ao longo do seu ciclo de vida, na redução de risco ambiental, poluição e outros impactos negativos da utilização dos recursos (incluindo o uso de energia), comparado a alternativas relevantes (Kemp \& Pearson, 2007, p. 7).

Como já assinalado acima, as quatro definições trabalhadas na revisão da literatura (eco inovação, inovação verde, inovação sustentável e inovação ambiental) apresentam pequenas diferenças na sua descrição. De maneira geral, as teorias abordam a inovação na perspectiva de novos ou modificados produtos, serviços, processos produtivos e de gestão, que oferecem benefícios ambientais. A questão ambiental é baseada prioritariamente na redução de emissões, resíduos e utilização de recursos naturais, reduzindo as externalidades negativas.

Essa perspectiva dialoga com a definição de eco inovação de Reid e Alasdair (2008), autores do painel europeu INNOVA:

[...[ significa a criação de novos bens com preços competitivos, processos, sistemas, serviços e procedimentos que satisfaçam necessidades humanas e tragam qualidade de vida para todas as pessoas com um ciclo de vida que minimiza a utilização de recursos naturais por unidade de saída, e minimiza a emissão de substâncias tóxicas (Reid \& Alasdair, 2008, p.7).

Revista de Administração e Inovação, São Paulo, v. 12, n.3 p. 226-250, jul./set. 2015. 
Schiederig et al. (2012) argumentam que pode haver alguma diferença entre os conceitos utilizados por pesquisadores, sendo que as teorias de eco inovação tendem a buscar uma análise do impacto por meio da avaliação do ciclo de vida, enquanto que as teorias de inovação verde ou ambiental tendem a fazer uma análise menos profunda. Os autores complementam que a intenção da redução pode ser tanto por motivações econômicas quanto ambientais. A Tabela 1 apresenta um resumo das principais diferenças das definições abordadas na revisão da literatura, sendo que a redução do impacto negativo sobre o meio ambiente, incluindo a redução de emissões, de resíduos e da utilização de recursos naturais são premissas comuns a todas as abordagens conceituais.

\section{Tabela 1}

Principais diferenças conceituais

\begin{tabular}{|c|l|}
\hline Inovação sustentável & $\begin{array}{l}\text { Abordagem de nichos tecnológicos; considera mudanças tecnológicas e } \\
\text { sociais concomitantemente. }\end{array}$ \\
\hline Inovação ambiental & $\begin{array}{l}\text { Orientada predominantemente por regulação ambiental; visa à redução de } \\
\text { custo; não aprofunda na análise do ciclo de vida. }\end{array}$ \\
\hline Eco inovação & $\begin{array}{l}\text { Considera o impacto e avaliação do ciclo de vida; inovação tecnológica, } \\
\text { social e institucional; prioriza o desempenho ambiental em detrimento à } \\
\text { meta ambiental. }\end{array}$ \\
\hline Inovação verde & $\begin{array}{l}\text { O desempenho da inovação considera o posicionamento da empresa e } \\
\text { imagem verde; não aprofunda na análise do ciclo de vida. }\end{array}$ \\
\hline
\end{tabular}

Fonte: Elaborado pelos autores, 2014.

Apesar das diferenças conceituais e de terminologias de iniciativas de inovação centrada em aspectos da sustentabilidade, alguns elementos da teoria de inovação embasam os condicionantes desse tipo de inovação, e são orientados pela oferta e/ou demanda de mercado, influencia política e institucional (Horbach, 2008), conforme descrito na Tabela 2.

\section{Tabela 2}

Determinantes da inovação sustentável

\begin{tabular}{|c|l|}
\hline $\begin{array}{c}\text { Impulsionado pela } \\
\text { oferta }\end{array}$ & $\begin{array}{l}\text { - compensações, incluindo redução de custo, originadas por inovações ambientais; } \\
\text { - características do mercado: tamanho da empresa e estrutura de mercado (um } \\
\text { monopólio pode restringir inovações sustentáveis pela ausência de incentivo); }\end{array}$ \\
& $\begin{array}{l}\text { - possibilidade de proteger as inovações (problemas em internalizar externalidades } \\
\text { positivas (spillovers) de uma inovação); atitude para risco e incerteza da inovação } \\
\text { ambiental; }\end{array}$ \\
& $\begin{array}{l}\text { - dependência e disponibilidade tecnológica (capital humano acumulado, } \\
\text { conhecimento disponível) induzem outras inovações; } \\
\text { - tempo disponível para realizar a inovação. }\end{array}$ \\
\hline $\begin{array}{c}\text { Impulsionado pela } \\
\text { demanda }\end{array}$ & $\begin{array}{l}\text { - demanda de mercado: governo, consumidores e empresas; } \\
\text { - consciência social da necessidade de uma produção mais limpa; consumo consciente } \\
\text { e preferencia por produtos sustentáveis. }\end{array}$ \\
\hline Influencias política & - política ambiental (instrumentos baseados em comando e controle ou incentivo de \\
\hline
\end{tabular}




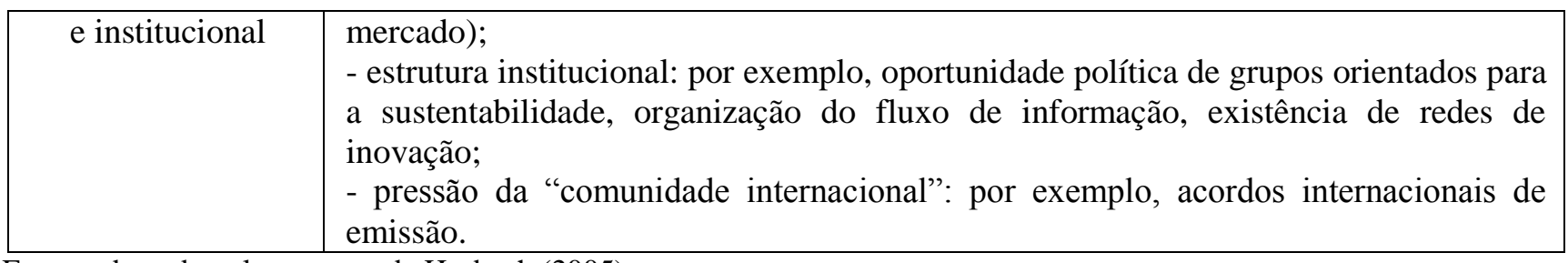

Fonte: adaptado pelos autores de Horbach (2005).

A influência do governo apresenta-se na literatura como um dos principais determinantes de projetos de inovação sustentável (Seiffert, 2007; Li, 2013). Além da compreensão dos determinantes, a análise do ambiente institucional, principalmente as condições políticas que favorecem a inovação sustentável, é relevante para o fomento de projetos de inovação sustentável no setor empresarial, visando ao desenvolvimento sustentável e à viabilização de iniciativas com foco na mitigação e adaptação aos impactos ambientais, especialmente aqueles relacionados com as mudanças climáticas.

\section{PROCEDIMENTOS METODOLÓGICOS}

A escolha do método bibliométrico para este estudo teve como objetivo apoiar a sistematização da produção científica sobre inovação sustentável de forma sistemática e aprofundada. O método possibilitou o levantamento e análise quantitativa da produção acadêmica, visando a um melhor entendimento conceitual sobre o tema. A análise das citações dos principais artigos buscou contribuir com futuros estudos por meio da indicação da influência de uma produção científica sobre outra e suas conexões intelectuais, além de possibilitar a comparação entre as diferentes teorias e autores.

\subsection{Bibliometria}

Bibliometria é um conjunto de leis e princípios empíricos que contribui para estabelecer os fundamentos teóricos da ciência da informação (Guedes \& Borschiver, 2005). Trata-se do estudo sobre os aspectos quantitativos de produção, disseminação e uso de uma informação, utilizando-se de modelos matemáticos e medidas para previsão e tomada de decisão (Tague-Sutckiffe, 1992). O autor descreve as três principais leis bibliométricas: Lotka, Zipf e Bradford. A Lei de Lotka baseia-se na produtividade de um determinado campo científico. Essa lei estabelece que o número de autores que fazem n contribuições em um campo científico é aproximadamente $1 / \mathrm{n} 2$, e aqueles que fazem uma única contribuição é de mais ou menos 60\% (Alvarado, 2002).

Revista de Administração e Inovação, São Paulo, v. 12, n.3 p. 226-250, jul./set. 2015. 
A classificação e a análise estatística das obras citadas pelos artigos são de grande auxílio aos pesquisadores para identificar linhas, teorias e conexões envolvidas (Moretti \& Campanario, 2009). Já a Lei de Zipf se baseia na medição da frequência do aparecimento das palavras em vários textos, possibilitando indexações. Por sua vez, a Lei de Bradford, a mais conhecida e utilizada, por meio da medição da produtividade de periódicos, indica a relevância de um periódico em um determinado campo de conhecimento.

Nesse estudo foram utilizadas as três leis, embora a Lei de Lotka o tenha sido parcialmente, por limite de espaço. A utilização do método bibliométrico pode contribuir de forma relevante para a sistematização do conhecimento sobre inovação sustentável, por meio de uma análise quantitativa da produção científica de um determinado período, possibilitando a identificação dos principais autores, artigos e periódicos relacionados ao tema (Moretti \& Campanario, 2009; Moran, 2010).

\subsection{Delineamento da pesquisa}

A Thomson Reuters Web of Knowledge foi a base de dados escolhida para este estudo, portanto, foram considerados apenas os periódicos e produções acadêmicas indexadas a essa base. Na fase inicial da coleta de dados, análise e revisão bibliográfica foram selecionadas as palavras-chave para formação do banco de dados e seleção dos artigos. A escolha das palavras-chave associadas ao campo de pesquisa é importante para guiar a busca pela literatura mais relevante e adequada. No decorrer da pesquisa, é necessário avaliar as palavras-chave para ampliar ou reduzir a busca, assim como incluir sinônimos (Collis \& Hussey, 2005).

A busca inicial foi realizada com as palavras-chave 'inovação sustentável'. Após realizar a revisão de alguns artigos foi possível identificar sinônimos adotados por alguns autores. Dessa maneira, foram incluídas outras palavras-chave como 'eco inovação', 'inovação verde' e 'inovação ambiental'.

Como a base de dados indexa artigos na língua inglesa, as expressões da Tabela 3 foram consideradas na busca pelos artigos.

\section{Tabela 3}

Palavras-chave do estudo bibliométrico

\begin{tabular}{|c|c|}
\hline Sustainable Innovation & Sustainable Innovations \\
\hline Sustainability Innovation & Sustainability Innovations \\
\hline Environment Innovation & Environment Innovations \\
\hline Environemental innovation & Environmental Innovations \\
\hline Green Innovation & Green Innovations \\
\hline
\end{tabular}

Fonte: elaborado pelos autores, 2013.

Revista de Administração e Inovação, São Paulo, v. 12, n.3 p. 226-250, jul./set. 2015. 
A Lei de Zipf foi aplicada para determinar as 20 palavras-chave do estudo bibliométrico. Por meio da revisão bibliográfica prévia foi possível verificar a frequência na ocorrência dessas palavraschave indexadas aos artigos do campo de conhecimento pesquisado.

A pesquisa obteve inicialmente 716 resultados sobre os quais foram adotados os seguintes critérios de refinamento: 1) limitou-se o período de busca entre 2008 e 2013; 2) analisaram-se apenas artigos científicos, considerando o rigor metodológico nesse tipo de publicação; 3) o domínio da pesquisa foi focado em ciências sociais, na área de business economics; 4) leitura dos resumos de todos os artigos, após o qual foram eliminados 32 artigos que não tinham aderência ao campo de conhecimento relacionado à inovação sustentável. Após este procedimento o resultado apresentou 110 artigos científicos que se tornaram o banco de dados (BD) da pesquisa.

$\mathrm{O}$ BD da pesquisa foi gerado a partir da ferramenta EndNote Web e exportado para uma planilha Microsoft Excel. Os artigos foram classificados por número de citação, e continham as seguintes informações: título do artigo e autor (es), periódico, ano da publicação, número total de citações no período pesquisado e média de citações por ano. Outros dados foram descartados, pois não eram relevantes para esse estudo.

Quanto à determinação da relevância dos artigos, foi considerado o número de citações, que é definido como informações obtidas de outras fontes com o objetivo de contribuir com as ideias desenvolvidas na obra (Martins \& Theóphilo, 2007).

\section{ANÁLISE DOS RESULTADOS}

A amostra analisada compreendeu 110 artigos científicos, publicados no período entre 2008 e abril de 2013, em 52 periódicos indexados à base de dados da Web of Knowledge. Ao todo, esses artigos receberam 638 citações na lista dos mais citados, que estão contidas em artigos indexados à mesma base de dados.

É importante destacar que os 20 artigos mais citados receberam 454 citações de outras publicações representam $18 \%$ da amostra pesquisada, e originaram mais de $70 \%$ do total das citações. Dessa maneira, foi possível identificar aderência à Lei de Lotka ou de 80/20, onde cerca de 20 das publicações representam 80 da circulação (Trueswell, 1969). 
A aplicação da Lei de Lotka mostrou que os 20 artigos analisados apresentaram 1.239 citações, incluindo artigos, anais de congressos, papers, teses, livros e relatórios. Os 20 artigos mais citados na produção científica analisada são detalhados na Tabela 4.

\section{Tabela 4}

Artigos mais citados

\begin{tabular}{|c|c|c|c|c|}
\hline$\#$ & Nome do artigo & $\mathbf{1}^{\circ}$ autor & Periódico & Citações \\
\hline 1 & $\begin{array}{c}\text { Strategic niche management and sustainable } \\
\text { innovation journeys: theory, findings, research } \\
\text { agenda, and policy }\end{array}$ & Schot, J. & $\begin{array}{l}\text { Tech Anal } \\
\text { Strategy }\end{array}$ & 67 \\
\hline 2 & $\begin{array}{l}\text { Determinants of environmental innovation - New } \\
\text { evidence from German panel data sources }\end{array}$ & Horbach, J. & Res Policy & 51 \\
\hline 3 & $\begin{array}{l}\text { Evolutionary approaches for sustainable innovation } \\
\text { policies: From niche to paradigm? }\end{array}$ & Nill, J. & Res Policy & 38 \\
\hline 4 & $\begin{array}{c}\text { Why sustainabilitity is now the key driver of } \\
\text { innovation }\end{array}$ & Nidumolu, R. & HBR & 29 \\
\hline 5 & $\begin{array}{l}\text { The driver of green innovation and green image - } \\
\text { Green core competence }\end{array}$ & Chen, Y. & J Bus Ethics & 28 \\
\hline 6 & $\begin{array}{l}\text { Information systems innovation for environmental } \\
\text { sustainability }\end{array}$ & Melville, N. & Mis Quart & 26 \\
\hline 7 & $\begin{array}{l}\text { What triggers environmental management and } \\
\text { innovation? Empirical evidence for Germany }\end{array}$ & Frondel, M. & Ecol Econ & 25 \\
\hline 8 & $\begin{array}{l}\text { Sectoral systems of environmental innovation: An } \\
\text { application to the French automotive industry }\end{array}$ & Oltra, V. & $\begin{array}{l}\text { Tech Forec } \\
\text { Soc }\end{array}$ & 23 \\
\hline 9 & $\begin{array}{l}\text { Agent-based modeling of the diffusion of } \\
\text { environmental innovations - An empirical approach }\end{array}$ & Schwarz, N. & $\begin{array}{l}\text { Tech Forec } \\
\text { Soc }\end{array}$ & 21 \\
\hline 10 & $\begin{array}{l}\text { Empirical influence of environmental management } \\
\text { on innovation: Evidence from Europe }\end{array}$ & Wagner, M. & Ecol Econ & 20 \\
\hline 11 & $\begin{array}{l}\text { Functions of innovation systems as a framework to } \\
\text { understand sustainable technological change: } \\
\text { Empirical evidence for earlier claims }\end{array}$ & Hekkert, M. & $\begin{array}{l}\text { Tech Forec } \\
\quad \text { Soc }\end{array}$ & 19 \\
\hline 12 & $\begin{array}{l}\text { Green and competitive? An empirical test of the } \\
\text { mediating role of environmental innovation strategy }\end{array}$ & Eiadat, Y. & J World Bus & 18 \\
\hline 13 & $\begin{array}{l}\text { Mainstreaming Green Product Innovation: Why and } \\
\text { How Companies Integrate Environmental } \\
\text { Sustainability }\end{array}$ & $\begin{array}{l}\text { Dangelico, R. } \\
\text { M. }\end{array}$ & J Bus Ethics & 14 \\
\hline 14 & $\begin{array}{l}\text { The effects of customer benefit and regulation on } \\
\text { environmental product innovation. Empirical } \\
\text { evidence from appliance manufacturers in Germany }\end{array}$ & Kammerer, D. & Ecol Econ & 14 \\
\hline 15 & $\begin{array}{c}\text { Environmental management systems and } \\
\text { technological environmental innovations: Exploring } \\
\text { the causal relationship }\end{array}$ & Ziegler, A. & Res Policy & 13 \\
\hline 16 & $\begin{array}{l}\text { The innovation effects of environmental policy } \\
\text { instruments - A typical case of the blind men and the } \\
\text { elephant? }\end{array}$ & Kemp, R. & Ecol Econ & 10 \\
\hline 17 & $\begin{array}{l}\text { Corporate Sustainability and Innovation in SMEs: } \\
\text { Evidence of Themes and Activities in Practice }\end{array}$ & $\begin{array}{l}\text { Bos- } \\
\text { Brouwers, H. } \\
\text { E. J. }\end{array}$ & $\begin{array}{l}\text { Bus Strateg } \\
\text { Environ }\end{array}$ & 10 \\
\hline 18 & $\begin{array}{l}\text { Regional innovation systems: development } \\
\text { opportunities from the 'green turn' }\end{array}$ & Cooke, $\mathrm{P}$. & $\begin{array}{l}\text { Tech Anal } \\
\text { Strategy }\end{array}$ & 10 \\
\hline 19 & $\begin{array}{l}\text { Adopting Sustainable Innovation: What Makes } \\
\text { Consumers Sign up to Green Electricity? }\end{array}$ & Ozaki, R. & $\begin{array}{l}\text { Bus Strateg } \\
\text { Environ }\end{array}$ & 9 \\
\hline
\end{tabular}


York, J.

J. Bus Venturing

Fonte: elaborado pelos autores a partir de informações do Web of Knowledge, 2013.

$\mathrm{Na}$ validação da amostra foram excluídas quatro referências em duplicidade de um mesmo artigo. Dessa forma, a amostra válida é de 1.235 citações originadas de 1.087 referências. Apenas 97 referências foram citadas duas ou mais vezes nos 20 artigos. O número de citações das referências é detalhado na Tabela 5 .

Tabela 5

Lista das citações nos 20 artigos analisados

\begin{tabular}{|c|c|c|c|c|}
\hline \# Citações & \# Artigos & \% & \# Citações & \% \\
\hline 1 & 990 & 91,1 & 990 & 80,2 \\
\hline 2 & 65 & 6,0 & 130 & 10,5 \\
\hline 3 & 20 & 1,8 & 60 & 4,9 \\
\hline 4 & 9 & 0,8 & 36 & 2,9 \\
\hline 5 & 1 & 0,1 & 5 & 0,4 \\
\hline 6 & 1 & 0,1 & 6 & 0,5 \\
\hline 7 & 0 & 0,0 & 0 & 0,0 \\
\hline 8 & 1 & 0,1 & 8 & 0,6 \\
\hline TOTAL & $\mathbf{1 0 8 7}$ & & $\mathbf{1 2 3 5}$ & \\
\hline
\end{tabular}

Fonte: Elaborado pelos autores, 2013.

A Lei de Lotka se aplica a esta amostra, já que muitos autores foram pouco citados e poucos foram muito citados. Neste caso, aqueles que obtiveram uma e duas incidências foram responsáveis por $90 \%$ das citações. Naturalmente uma amostra maior seria preferível, mas esta já foi suficiente para mostrar a concentração proposta pela referida lei. Este resultado sugere que os pesquisadores devem atentar para os autores que são muito citados a fim de compreender as linhas mestras do campo de estudo. Não é prudente negligenciar os autores pouco citados, pois representam um esforço de pesquisa despendido pelos mesmos e, sem dúvida, uma opção às linhas dominantes.

Em seu estudo Moretti e Campanario (2009) já haviam observado que a expressiva produção apresentada no Encontro da Associação Nacional de Pós-graduação e Pesquisa em Administração (EnANPAD), caso do estudo, em sua maioria, não foi aproveitada nos anos seguintes. Sobre este aspecto os autores dizem:

Existe de fato uma concentração - como a tabela anterior demonstrou - em poucos autores de teoria geral da administração ou de estratégia. Essa é uma indicação de que a forma como os trabalhos são desenvolvidos contribui reduzidamente para o avanço desse campo, por terem suas ideias já assimiladas e difundidas. Há um domínio da reprodução das mesmas ideias, reforçando a tese de zona de conforto intelectual (Moretti \& Campanario, 2009, p. 79). 
Inovação sustentável: uma perspectiva comparada da literatura internacional e nacional

A lista das 97 produções acadêmicas que receberam duas ou mais citações é detalhada na Tabela 6 a seguir. 


\section{Tabela 6}

Lista das obras com três ou mais citações na amostra pesquisada

\begin{tabular}{|c|c|}
\hline \# & AUTOR e ARTIGO \\
\hline 8 & $\begin{array}{l}\text { Rehfeld, K., \& Rennings, K., Ziegler, A. (2007). Integrated product policy and environmental } \\
\text { product innovations: an empirical analysis. Ecological Economics 61, 91-100. }\end{array}$ \\
\hline 6 & $\begin{array}{l}\text { Porter, M., \& Van der Linde, C. (1995). Toward a new conception of the environment- } \\
\text { competitiveness relationship. Journal of Economic Perspectives } 9(4), 97-118 \text {. }\end{array}$ \\
\hline 5 & $\begin{array}{l}\text { Rennings, K. (2000). Redefining innovation - eco-innovation research and the contribution } \\
\text { from ecological economics. Ecological Economics } 32(2), 319-332 .\end{array}$ \\
\hline 4 & $\begin{array}{l}\text { Brunnermeier, S.B., \& Cohen, M.A. (2003). Determinants of environmental innovation in US } \\
\text { manufacturing industries. Journal of Environmental Economics and Management 45, 278-293. }\end{array}$ \\
\hline 4 & $\begin{array}{l}\text { Frondel, M., Horbach, J., \& Rennings, K. (2007). End-of-pipe or cleaner production? An } \\
\text { empirical comparison of environmental innovation decisions across OECD countries. Business } \\
\text { Strategy and the Environment, 16(8), 571-584. }\end{array}$ \\
\hline 4 & Greene, W.H. (2000). Econometric Analysis, Fourth edition. Prentice-Hall, Inc., London. \\
\hline 4 & $\begin{array}{l}\text { Hemmelskamp, J. (1999). Der einfluss der umweltpolitik auf das innovationsverhalten - Eine } \\
\text { oekonometrische Untersuchung. Zeitschrift fuer Umweltpolitik \& Umweltrecht 1, 33-66. }\end{array}$ \\
\hline 4 & $\begin{array}{l}\text { Jaffe, A.B., Newell, R.G., \& Stavins, R.N. (2002) Environmental policy and technological } \\
\text { change, Environ. Resour. Econ. } 22(1-2) 41-69 .\end{array}$ \\
\hline 4 & $\begin{array}{l}\text { Jaffe, A., \& Palmer, K. (1997). Environmental regulation and innovation: a panel study. The } \\
\text { Review of Economics and Statistics X, 610-619. }\end{array}$ \\
\hline 4 & $\begin{array}{l}\text { Nelson R.R., \& Winter, S.G. (1982). An evolutionary theory of economic change, The Belknap } \\
\text { Press of Harvard University Press. }\end{array}$ \\
\hline 4 & $\begin{array}{l}\text { Porter, M., \& van der Linde, C. (1995). Green and competitive: ending the stalemate, Harvard } \\
\text { Business Review September-October, 120-133. }\end{array}$ \\
\hline 4 & $\begin{array}{l}\text { Rennings, K., Ziegler, A., Ankele, K., \& Hoffmann, E. (2006). The influence of different } \\
\text { characteristics of the EU environmental management and auditing scheme on technical } \\
\text { environmental innovations and economic performance. Ecological Economics 57 (1), 45-59. }\end{array}$ \\
\hline 3 & $\begin{array}{l}\text { Dasgupta, S., Hettig, H., \& Wheeler, D. (2000). What improves environmental compliance? } \\
\text { Evidence fromMexican industry. Journal of Environmental Economics and Management 39, } \\
\text { 39-66. }\end{array}$ \\
\hline 3 & $\begin{array}{l}\text { Dosi G., (1982). Technological paradigms and technological trajectories, Res. Policy } 11 \text { (3) } \\
\text { 147-162. }\end{array}$ \\
\hline 3 & Freeman, C., \& Soete, L. (1999). The economics of industrial innovation. London: Pinter. \\
\hline 3 & $\begin{array}{l}\text { Frondel, M., Horbach, J., \& Rennings, K. (2008). What triggers environmental management } \\
\text { and innovation? Empirical evidence for Germany. Ecological Economics, 66(1), 153-160. }\end{array}$ \\
\hline 3 & $\begin{array}{l}\text { Geels F.W. (2002). Technological transitions as evolutionary reconfiguration processes: a } \\
\text { multi-level perspective and a case-study, Res. Policy } 31(8-9) 1257-1274 \text {. }\end{array}$ \\
\hline 3 & $\begin{array}{l}\text { Hair, J. F., Anderson, R. E., Tatham, R. L., \& Black, W. C. (1998). Multivariate data analysis. } \\
\text { New Jersey: Prentice-Hall International, Inc. }\end{array}$ \\
\hline 3 & $\begin{array}{l}\text { Hart, S. L. (1995). A natural-resource-based view of the firm. Academy of Management } \\
\text { Review, 20(4): 986-1014. }\end{array}$ \\
\hline 3 & $\begin{array}{l}\text { Horbach J. (2008). Determinants of environmental innovation - new evidence from German } \\
\text { panel data sources, Res. Policy } 37 \text { (1) } 163-173 .\end{array}$ \\
\hline 3 & $\begin{array}{l}\text { Kemp R. (1997). Environmental Policy and Technical Change: A Comparison of the } \\
\text { Technological Impact of Policy Instruments, Edward Elgar Publishing. }\end{array}$ \\
\hline 3 & $\begin{array}{l}\text { Loorbach, D. (2007). Transition management: new mode of governance for sustainable } \\
\text { development, PhD thesis, Erasmus University Rotterdam. }\end{array}$ \\
\hline 3 & $\begin{array}{l}\text { Pavitt, K. (1984). Sectoral patterns of technical change: towards a taxonomy and a theory. } \\
\text { Research Policy } 13(6), 343-373 \text {. }\end{array}$ \\
\hline 3 & $\begin{array}{l}\text { Rip, A., \& Kemp, R. (1998). Technical change. In: Rayner, S., Majone, E.L. (Eds.), Human } \\
\text { Choice and Climate Change. Batelle Press, Columbus, OH, pp. 327-399. }\end{array}$ \\
\hline 3 & Rotmans J., R. Kemp, \& M. Van Asselt. (2001). More evolution than reve \\
\hline
\end{tabular}

Revista de Administração e Inovação, São Paulo, v. 12, n.3 p. 226-250, jul./set. 2015. 


\begin{tabular}{|c|l|}
\hline & management in public policy, Foresight 3 (1) 15-31. \\
\hline 3 & $\begin{array}{l}\text { Russo, M.V., \& Fouts, P.A. (1997). A resource-based perspective on corporate environmental } \\
\text { performance and profitability. Academy of Management Journal 40 (3), 534-559. }\end{array}$ \\
\hline 3 & $\begin{array}{l}\text { Sharma, S., \& Vredenburg, H. (1998). Proactive corporate environmental strategy and the } \\
\text { development of competitively valuable organizational capabilities. Strategic Management } \\
\text { Journal 19 (8), 729-753. }\end{array}$ \\
\hline 3 & $\begin{array}{l}\text { Shrivastava, P. (1995). Environmental technologies and competitive advantage. Strategic } \\
\text { Management Journal 16, 183-200. }\end{array}$ \\
\hline 3 & $\begin{array}{l}\text { Van der Laak, W.W.M., Raven, R.P.J.M., \& Verbong, G.P.J. (2007). Strategic niche } \\
\text { management for biofuels: analysing past experiments for developing new biofuels policies. } \\
\text { Energy Policy 35, 3213-3225. }\end{array}$ \\
\hline 3 & $\begin{array}{l}\text { WCED (World Commission on Environment and Development). (1987). Our Common Future, } \\
\text { Oxford, UK: Oxford University Press. }\end{array}$ \\
\hline 3 & $\begin{array}{l}\text { Wernerfelt, B. (1984). A resource-based view of the firm. Strategic Management Journal 5, } \\
\text { 171-180. }\end{array}$ \\
\hline $\begin{array}{l}\text { Ziegler, A., \& Rennings, K. (2004) Determinants of environmental innovations in Germany: do } \\
\text { organizational measures matter? A discrete choice analysis at the firm level. Discussion Paper } \\
\text { 04-30. ZEW, Mannheim. }\end{array}$ \\
\hline
\end{tabular}

Fonte: elaborado pelos autores, 2013.

Com base na análise das 97 publicações que obtiveram duas ou mais citações, foi possível identificar que 67 foram artigos publicados em periódicos internacionais, 19 eram livros e 11 foram classificados como outros, incluindo teses, papers e relatórios. Na Tabela 7 encontram-se os títulos dos periódicos onde foram publicados os 67 artigos científicos analisados, o número de artigos por periódico, fator de impacto, país e percentual.

\section{Tabela 7}

Lista dos periódicos por publicação

\begin{tabular}{|l|l|l|l|l|}
\hline Periódicos & $\begin{array}{l}\# \\
\text { citações }\end{array}$ & FI & País & $\%$ \\
\hline Ecological Economics & 8 & 2.855 & Holanda & 11,94 \\
\hline Research Policy & 8 & 2.850 & Holanda & 11,94 \\
\hline $\begin{array}{l}\text { Journal of Environmental Economics and } \\
\text { Management }\end{array}$ & 5 & 1.969 & EUA & 7,5 \\
\hline Academy of Management Journal & 4 & 5.906 & EUA & 6,0 \\
\hline Business Strategy and the Environment & 4 & 3.236 & EUA & 6,0 \\
\hline Strategic Management Journal & 4 & 3.367 & EUA & 6,0 \\
\hline Harvard Business Review & 2 & 1.519 & EUA & 3,0 \\
\hline Journal of Business Ethics & 2 & 1.270 & Holanda & 2,99 \\
\hline Journal of Evolutionary Economics & 2 & 0.723 & Alemanha & 3,0 \\
\hline Academy of Management Executive & 1 & nd & nd & 1,5 \\
\hline Academy of Management Review & 1 & 7.895 & EUA & 1,5 \\
\hline Administrative Science Quarterly & 1 & 4.182 & EUA & 1,5 \\
\hline American Economic Review & 1 & 2.792 & EUA & 1,5 \\
\hline American Sociological Review & 1 & 4.077 & EUA & 1,5 \\
\hline California Management Review & 1 & 1.667 & EUA & 1,5 \\
\hline Energy Policy & 1 & 2.743 & Inglaterra & 1,5 \\
\hline Environmental \& Resource Economics & 1 & 1.795 & Holanda & 1,49 \\
\hline
\end{tabular}




\begin{tabular}{|l|l|l|l|l|}
\hline Foresight & 1 & nd & Inglaterra & 1,5 \\
\hline Futures & 1 & 1.111 & Inglaterra & 1,5 \\
\hline Harvard Environmental Law Review & 1 & 1.607 & EUA & 1,5 \\
\hline Industrial and Corporate Change & 1 & 1.331 & Inglaterra & 1,5 \\
\hline International Journal of Technology Management & 1 & 0.564 & Suíça & 1,5 \\
\hline Journal of Economic Literature & 1 & 6.667 & EUA & 1,5 \\
\hline Journal of Economic Perspectives & 1 & 3.489 & EUA & 1,5 \\
\hline Land Economics & 1 & 1.261 & EUA & 1,5 \\
\hline Management Science & 1 & 1.859 & EUA & 1,5 \\
\hline Organization Science & 1 & 3.351 & EUA & 1,5 \\
\hline Organization Studies & 1 & 2.190 & Inglaterra & 1,5 \\
\hline Organizational Behavior and Human Decision & 1 & 2.816 & EUA & 1,5 \\
Processes & & & & \\
\hline Policy Sciences & 1 & 1.059 & Holanda & 1,49 \\
\hline Production and Operations Management & 1 & nd & EUA & 1,5 \\
\hline R \& D Management & 1 & 1.580 & Inglaterra & 1,5 \\
\hline Review of Economics and Statistics & 1 & 2.346 & EUA & 1,5 \\
\hline Scientific American & 1 & nd & EUA & 1,5 \\
\hline Technological Forecasting and Social Change & 1 & 2.106 & EUA & 1,5 \\
\hline Technology Analysis \& Strategic Management & 1 & 1.095 & Inglaterra & 1,5 \\
\hline Technovation & 1 & 3.177 & Holanda & 1,49 \\
\hline TOTAL & $\mathbf{6 7}$ & & & $\mathbf{1 0 0}$ \\
\hline
\end{tabular}

Fonte: elaborado pelos autores a partir de dados do JCR Social Sciences, 2012.

Os artigos da Tabela 7 foram publicados em 37 periódicos internacionais, sendo que desses, 33 artigos foram publicados em apenas seis periódicos. A análise evidencia que cerca de $50 \%$ dos artigos foram publicados por $16 \%$ periódicos na amostra estudada. Nesse sentido, há indícios de que alguns periódicos apresentam maior interesse em publicar artigos científicos relacionados ao tema sustentabilidade, incluindo Ecological Economics, Research Policy, Journal of Environmental Economics and Management, Academy of Management Journal, Business Strategy and the Environment e Strategic Management Journal.

Com relação ao fator de impacto (FI) dos periódicos, cinco dos seis periódicos que publicaram mais artigos apresentam FI acima de 2,8, chegando a 5,9. Dos periódicos analisados, 13 apresentam FI acima de 2,8, tendo publicado 53,7\% das citações analisadas. Nesse sentido, há indícios de que a temática inovação sustentável é relevante para periódicos que possuem considerável índice de FI.

Analisando a produção por país, a Holanda se destacou. Os periódicos holandeses Ecological Economics e Research Policy foram os que mais publicaram artigos relacionados ao tema, representando 23,9\% da produção. Ambas as publicações pertencem à holandesa Elsevier, uma das maiores e principais editoras do mundo focadas na área acadêmica. Essa pode ser uma das razões do destaque da Holanda na produtividade. Outro fator a considerar é que importantes pesquisadores que publicam artigos na temática estudada são de países europeus. 
Apesar do destaque da Holanda, os Estados Unidos lideram a produção, com 52,2\% em 21 periódicos. A produção americana foi mais dispersa que a holandesa em número de periódicos. O Gráfico 1 mostra a posição dos países e sua participação na produção.

\section{Gráfico 1}

Produção por país

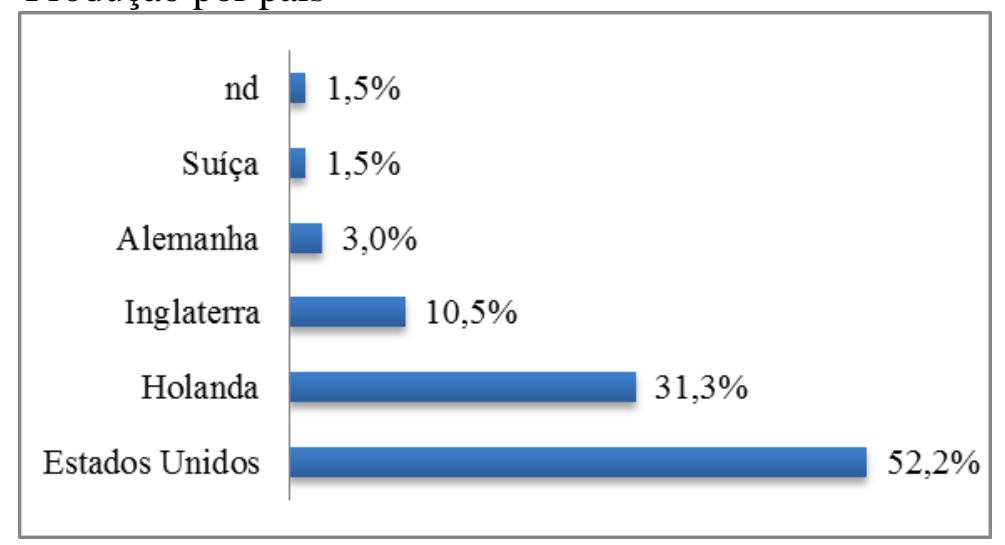

Fonte: Elaborado pelos autores, 2013.

\section{PRODUÇÃO ACADÊMICA BRASILEIRA}

A produção acadêmica sobre inovação sustentável ainda é limitada no Brasil. Na pesquisa detalhada acima não foi identificado nenhum artigo originado de pesquisadores do Brasil, conforme parâmetros de refinamento adotados. Dessa forma, nova pesquisa foi realizada na base Web of Knowledge em junho de 2014, considerando os mesmos aspectos de refinamento de busca detalhando no capítulo de metodologia. Foram encontrados 74 artigos indexados às palavras-chave da pesquisa, sem delimitação de período de publicação.

Após validação do banco de dados, apenas oito artigos foram considerados aderentes ao tema inovação sustentável. Dessa amostra, apenas três artigos receberam citações, conforme demonstrado na Tabela 8.

\section{Tabela 8}

Lista de artigos de pesquisadores brasileiros na amostra pesquisada

\begin{tabular}{|c|c|c|c|c|}
\hline$\#$ & Nome do artigo & $\mathbf{1}^{\mathbf{0}}$ autor & Periódico & Citações \\
\hline 1 & $\begin{array}{c}\text { Innovation and sustainability: new models and } \\
\text { propositions }\end{array}$ & Barbieri, J. C. & RAE & 10 \\
\hline 2 & $\begin{array}{c}\text { A emergência da tecnologia social: revisitando o } \\
\text { movimento da tecnologia apropriada como } \\
\text { estratégia de desenvolvimento sustentável }\end{array}$ & Rodrigues, I. & RAP & 6 \\
\hline 3 & $\begin{array}{c}\text { Inovação e sustentabilidade na produção de } \\
\text { energia: o caso do sistema setorial de energia } \\
\text { eólica no Brasil }\end{array}$ & $\begin{array}{c}\text { Nascimento, T. } \\
\text { C. }\end{array}$ & $\begin{array}{c}\text { Cadernos } \\
\text { EBAPE.BR }\end{array}$ & 1 \\
\hline
\end{tabular}

Revista de Administração e Inovação, São Paulo, v. 12, n.3 p. 226-250, jul./set. 2015. 
Fonte: elaborado pelos autores, 2014.

O primeiro artigo brasileiro sobre o tema foi publicado em 2008, sendo que a primeira citação ocorreu em 2010 em um artigo publicado no mesmo ano. O artigo mais relevante, com 10 citações, é do Prof. Barbieri. Ao todo, os três artigos receberam apenas 17 citações até a presente data.

As evidências demonstram que a produção acadêmica sobre inovação sustentável no Brasil ainda é incipiente e recente. Nova pesquisa realizada no Google Acadêmico indica que gradativamente a produção sobre o tema tem sido ampliada, embora diversos dos estudos encontrados devam ainda ser considerados em fase de elaboração, pois foram publicados apenas em anais de eventos.

Foram encontrados estudos em diversos setores, incluindo o eletroeletrônico (Pereira et al, 2013; Scandelari \& Cunha, 2013), petroquímico (Giovannini \& Kruglianskas, 2008; Brock e Sá, 2011), cosmético (Curi, Junqueira, Bertoni, Camargo \& Curi, 2010), sucroenergético (Carvalho \& Barbieri, 2010), agronegócio (Oliveira \& Ipiranga, 2011; Freitas et al, 2012), bens de consumo (Medeiros, Ribeiro \& Cruz, 2012; Pinsky, Dias \& Kruglianskas, 2013), químico (Menezes et al, 2013), além de artigos de natureza conceitual (Ladeira, Costa, Berte \& Falcão, 2010; Gonçalves-Dias et al, 2012; Sehnem, Lazzarotti, Puffal \& Cericato, 2013). No entanto, a maioria das pesquisas publicadas no Brasil é qualitativa, baseadas em estudos de caso ou ensaios teóricos, impossibilitando a generalização dos resultados (Yin, 2005).

\section{CONSIDERAÇÕES FINAIS}

O objetivo desse estudo foi realizar uma pesquisa bibliográfica sobre o conceito de inovação sustentável. Para isso foi utilizado o método bibliométrico com o intuito de facilitar o levantamento e seleção dos artigos científicos relevantes nessa temática.

Foi analisada quantitativamente a produção acadêmica no período entre os anos de 2008 e abril de 2013, e outra em junho de 2014 centrada apenas na produção brasileira. Foram identificados os autores mais citados na base Web of Knowledge e verificado se os periódicos que publicaram suas obras estão entre os melhores classificados por fator de impacto. A amostra analisada compreendeu 110 artigos científicos, em 52 periódicos indexados à base de dados da Web of Knowledge. Ao todo, esses artigos receberam 638 citações, que estão contidas em artigos indexados à mesma base de dados.

Uma utilização parcial da Lei de Zipf, considerando apenas as palavras-chave, mostrou uma falta de padronização das diferentes terminologias para definir o que vem a ser inovação sustentável. A literatura, em geral, foca no conceito de inovação para a redução do impacto negativo sobre o meio

Revista de Administração e Inovação, São Paulo, v. 12, n.3 p. 226-250, jul./set. 2015. 
ambiente, utilizando terminologias como 'verde', 'eco', 'ambiental' e 'sustentável' conforme já havia sido postulado por Schiederig et al (2012).

A Lei de Lotka se aplicou a esta amostra, já que os resultados mostraram que uma pequena parcela dos autores foi muito citada, enquanto que $90 \%$ das citações pertencem aos que tiveram apenas uma e duas citações. Este resultado está de acordo com Moretti e Campanario (2009) que fizeram um estudo com uma amostra maior de artigos nos anais do EnAnpad.

A aplicação da Lei de Bradford mostrou indícios de relevância dos periódicos internacionais com relação ao tema inovação sustentável. A novidade reside no fato de que as publicações da Holanda estarem no mesmo nível dos Estados Unidos quanto ao quesito de maior volume de produção sobre o tema. Entretanto, deve-se considerar que os dois periódicos que mais publicaram artigos relacionados ao tema são holandeses e pertencentes à Elsevier, também holandesa. Essa pode ser uma das razões do destaque da Holanda na produtividade, além do fato de importantes pesquisas terem sido conduzidas em países europeus.

Os periódicos que apresentaram o maior número de artigos publicados sobre o assunto, e que supostamente são aqueles de maior relevância na área de sustentabilidade, foram: Ecological Economics, Research Policy, Journal of Environmental Economics and Management, Academy of Management Journal, Business Strategy and the Environment e Strategic Management Journal.

Dos 37 periódicos analisados, 13 apresentam FI acima de 2,8, tendo publicado 53,7\% das citações analisadas. Nesse sentido, há indícios de que a temática inovação sustentável é relevante para periódicos que possuem considerável índice de FI.

A produção acadêmica brasileira sobre o tema pesquisado ainda é incipiente, mas gradativamente vem aumentando nos últimos anos.

As contribuições deste estudo para o campo acadêmico são diversas. Em primeiro lugar, como auxílio aos pesquisadores que desejam conduzir pesquisas bibliográficas de qualidade para seus trabalhos. Em segundo, pode-se estabelecer camadas de aprofundamento das análises de citações até se encontrar relações entre as linhas mestras nas quais o campo se apoia. E por fim, pode-se relacionar a produtividade dos autores analisando as coautorias dos trabalhos.

As principais contribuições deste estudo foram: a revisão da literatura sobre inovação sustentável e a demonstração prática da utilização do método bibliométrico com o intuito de facilitar o levantamento e seleção dos artigos científicos. Espera-se pode ter contribuído para que futuros pesquisadores possam aperfeiçoar seus métodos de pesquisa bibliográfica desenvolvendo trabalhos com melhor embasamento dos estudos de ponta. 
Como recomendação para pesquisas futuras, o aprofundamento nas análises das citações mencionado acima possibilitará um amplo mapa do conhecimento do campo na inovação sustentável. Além disso, uma análise aprofundada da produção acadêmica brasileira possibilitará identificar as conexões acadêmicas, indicar lacunas de conhecimento e oportunidades de pesquisas setoriais sobre a temática.

\section{REFERÊNCIAS}

Aligleri, L., Aligleri, L. A., \& Kruglianskas, I. (2009). Gestão socioambiental: responsabilidade $e$ sustentabilidade do negócio. São Paulo: Atlas.

Alvarado, U. R. (2002). A lei de Lotka na bibliometria brasileira. Ciência da Informação, Brasília, v. 31, n 2, p. 14-20, maio/ago.

Brock, V. F., \& Sá, U. G. C. (2011). Evolução das práticas organizacionais: um estudo da gestão ambiental e inovação sustentável numa indústria petroquímica de triunfo, RS. In: V Encontro de Estudos em Estratégia ANPAD. Porto Alegre.

Carvalho, A. P., \& Barbieri, J. C. (2010). Innovation for sustainability: overcoming the productivity of the sugar and ethanol industry's conventional system. Journal of Technology management \& Innovation, v. 5 (4), p. 83-94.

Chen, Y (2008). The driver of green innovation and green image-green core competence. Journal of Business Ethics, v. 81 (3), p. 531-543.

Chen, Y., Lai, S., \& Wen, C (2006). The influence of green innovation performance on corporate advantage in Taiwan. Journal of Business Ethics, v. 67 (4), p. 331-339.

Collis, J., \& Hussey, R (2005). Pesquisa em administração: um guia prático para alunos de graduação e pós-graduação. $2^{\mathrm{a}}$ ed. Porto Alegre: Bookman.

Curi, D. P, Junqueira, E. A., Bertoni, E., Camargo, E., \& Almeida, M. C. M. (2010). Inovação Sustentável nas Empresas de Cosméticos. In: XXXIV Encontro da ANPAD. Rio de Janeiro: Associação Nacional de Pós-Graduação e Pesquisa em Administração.

Elkington, J. (1998). Partnerships from cannibals with forks: The triple bottom line of 21st-century business. Environmental Quality Management, v. 8 (1), pp. 37-51.

(2001). Canibais com garfo e faca. Makron Books.

Freitas, C. C. G. et al. (2012). Transferência tecnológica e inovação por meio da sustentabilidade. Revista de Administração Pública, v. 46 (2), p. 363-384. 
Frondel, M., Horbach, J., \& Rennings, K. (2008). What triggers environmental management and innovation? Empirical evidence for Germany. Ecological Economics, v. 66, pp. 153-160.

Giovannini, F., \& Kruglianskas, I. (2008). Fatores críticos de sucesso para a criação de um processo inovador sustentável de reciclagem: um estudo de caso. Revista de Administração Contemporânea, 12 (4), p. 931-951.

Gonçalves-Dias, S. L. F., Guimaraes, L. F., \& dos Santos, M. C. L. (2012). Inovação no desenvolvimento de produtos "verdes": integrando competências ao longo da cadeia produtiva. Revista de Administração e Inovação, v. 9 (3), 129-153.

Guedes, V., \& Borschiver, S. (2005). Bibliometria: uma ferramenta estatística para a gestão da informação e do conhecimento, em sistemas de informação, de comunicação e de avaliação científica e tecnológica. In: CINFORM - Encontro Nacional de Ciência da Informação VI, v. 6, dezembro.

Hart, S.L., \& Milstein, M. B (2004). Criando valor sustentável. RAE Executivo, v. 3 (2), maio/julho.

Horbach, J. (2005). Indicator systems for sustainable innovation. Heidelberg, Germany: PhysicaVerlag.

Kemp, R, \& Pontoglio, S. (2011). The innovation effects of environmental policy instruments - a typical case of the blind men and the elephant? Ecological Economics, v. 72, pp. 28-36.

Kemp, R., \& Pearson, P. (2007). Final report of the MEI project measuring eco innovation. UM Merit. Disponível em: http://www.merit.unu.edu/MEI/deliverables/MEI\%20D15\%20Final\%20report\%20about\%20measur ing\%20eco-innovation.pdf. Acesso em: 25/6/2013.

Kiron, D. et al. The innovation bottom line. MIT Sloan Management Review Research Report. Winter, 2013.

Ladeira, W. J., Costa, J. C., Berte, R. S., Falcão, C. A. (2010). Antecedentes da inovação sustentável: uma investigação através da modelagem de equações estruturais. In: XXVI Simpósio de Gestão da Inovação Tecnológica ANPAD. Vitória: Associação Nacional de Pós-Graduação e Pesquisa em Administração.

Martins, G. A., \& Theóphilo, C. R (2007). Metodologia na investigação científica para ciências sociais aplicadas. São Paulo: Atlas.

May, P. H., Lustosa, M. C., \& Vinha, V. Orgs. (2003). Economia do meio ambiente: teoria e prática. Rio de Janeiro: Elsevier.

Medeiros, J. F. D., Ribeiro, J. L. D., \& Cruz, C. M. L. (2012). Environmentally sustainable innovation and success factors in the perception of managers from the manufacturing industry. Cadernos EBAPE.BR, 10 (3), 652-676.

MORAN, M. R. et al (2010). Alianças estratégicas: uma análise bibliométrica da produção cinetífica entre 1989 e 2008. Revista de Ciências da Administração, v. 12 (27), p. 42-62, maio/agosto. 
Moretti, S. L. A., \& Campanario, M. A (2009). A produção intelectual brasileira em responsabilidade social empresarial - RSE, sob a ótica da bibliometria. Revista de Administração Contemporânea, $v$. 13, edição especial, art. 5, p. 68-86, junho.

Nações Unidas (1987). Relatório Brundtland: Nosso Futuro Comum. Disponível em: http://www.onu.org.br/a-onu-em-acao/a-onu-e-o-meio-ambiente. Acesso em: 30/9/2012.

Nidumolu, R., Prahalad, K., \& Rangaswami, M. R. (2009). Why sustainability is now the key driver of innovation. Harvard Business Review, v. 87 (9), pp. 56-64.

Oliveira, L. G. L., \& Ipiranga, A. S. R. (2011). Evidences of the sustainable innovation in the cashew agribusiness context in Ceará-Brazil. Revista de Administração Mackenzie, v. 12 (5), p. 122-150.

Oltra, V., \& Saint Jean, M. (2009). Sectoral systems of environmental innovation: an application to the French automotive industry. Technological Forecasting and Social Change, v. 76 (4), pp. 567-583.

OSLO, Manual (2004). Proposta de diretrizes para coleta e interpretação de dados sobre inovação tecnológica. Tradução da Financiadora de Estudos e Projetos. Paris: Organization for Economic Cooperation and Development.

PEREIRA, D. C. et al. (2013). A influência da inovação sustentável na avaliação de alternativas de compra pelo consumidor de produtos eletroeletrônicos. In: XXXVII Encontro da ANPAD. Rio de Janeiro: Associação Nacional de Pós-Graduação e Pesquisa em Administração.

Pinsky, V. C., Dias, J. L., \& Kruglianskas, I. (2013). Gestão estratégica da sustentabilidade e inovação. Revista de Administração da UFSM, v. 6 (3), p. 465-480.

PINTEC. Pesquisa em Inovação 2011. (2013). Rio de Janeiro: Instituto Brasileiro de Geografia e Estatística. em: http://www.pintec.ibge.gov.br/downloads/pintec2011\%20publicacao\%20completa.pdf. Acesso em: $7 / 4 / 2014$.

Porter, M. E., \& Van der Linde, C. (1995a) Green and competitive: ending the stalemate. Harvard Business Review, v. 73 (5), p. 120-134, September/October.

(1995b) Toward a new conception of the environment-competitiveness relationship. The Journal of Economic Perspectives, v. 9 (4), pp. 97-118.

Reid, A., \& Miedzinski, M. (2008). Sectoral innovation watch in Europe/Eco-innovation. Brussels: Europe Innova.

Rennings, K. (2000). Redefining innovation-eco-innovation research and the contribution from Ecological Economics. Ecological Economics, v. 32 (2), pp. 319-332.

Scandelari, V. D. R. N., \& Cunha, J. C. D. (2013). Ambidextrality and the socioenvironmental performance of companies in the electro-electronic sector. Revista de Administração de Empresas, v. 53 (2), p. 183-198.

Schaltegger S., \& Wagner, M. (2011). Sustainable entrepreneurship and sustainability innovation: categories and interactions. Business Strategy and the Environment, v. 20 (4), pp. 222-237, May. 
Schiederig, T., Tietze, F., \& Herstatt, C. (2012) Green innovation in technology and innovation management - an exploratory literature review. R\&D Management, v. 42 (2), pp. 180-192.

SDSN - Sustainable Development Solutions Network - Uma agenda de ação para o desenvolvimento sustentável. (2013). Relatório para o secretário geral da ONU. Disponível em: http://unsdsn.org/files/2013/06/130619-Uma-Agenda-de-A\%C3\%A7\%C3\%A3o-Para-ODesenvolvimento-Sustent\%C3\%A1vel-US-LETTER.pdf. Acesso em: 24/6/2013.

Sehnem, S., Lazzarotti, F., Puffal, D. P., \& Cericato, A. (2013). Inovação sustentável: autorias e temas estudados. In: XXXVII Encontro da ANPAD. Rio de Janeiro: Associação Nacional de PósGraduação e Pesquisa em Administração.

Tague-Sutckiffe, J. (1992). An introduction to informetrics. Information Processing \& Management, $v$. $28(1)$, pp. 1-3.

Tidd, J., \& Bessant, J. (2009). Managing innovation: integrating technological, market and organizational change. 4 ed. Wiley.

Trueswell, R. W. (1969). Some behavioral patterns of library users: the 80/20 rules. Wilson Library Bulletin, [s.l.], v. 43 (5), p. 458-461.

UN. United Nations Sustainable Development Knowledge Platform. Disponível em: http://sustainabledevelopment.un.org/index.html. Acesso em: 2/12/2014.

WBCSD. (2010). World Business Council for Sustainable Development. Visão 2050: a nova agenda para as empresas. Disponível em: $\quad$ http://www.wbcsd.org/vision2050.aspx. Acesso em: 24/6/2013.

Yin, R. K. (2005). Estudo de caso: planejamento e métodos. Trad. Daniel Grassi. $3^{\text {a }}$ ed. Porto Alegre: Bookman. 


\title{
SUSTAINABLE INNOVATION: A PERSPECTIVE OF COMPARATIVE INTERNATIONAL AND NATIONAL LITERATURE
}

\begin{abstract}
Innovation is one of the main factors that positively influence the competitiveness and the economic development. Sustainable innovation is presented as an alternative to building a new low-carbon economy, aimed at the sustainable development and the viability of initiatives focusing on mitigation and adaptation of environmental impacts, especially those related to climate change. The aim of this study was to conduct a literature review on sustainable innovation. The bibliometric method was used in order to map and identify the relevant scientific production in that field. The international academic production in the period between 2008 and April 2013 and the Brazilian academic production until June 2014 were analyzed. The major results include the lack of standardized terminology and conceptual consensus on sustainable innovation, the incipient Brazilian production and the fact that journals from Netherlands and the United States concentrate the highest production volume on the subject. The major contribution of the study are: to assist scholars with a state-of-art literature review on sustainable innovation, and highlight the importance to consider relevant authors and journals that are in the frontier of the knowledge to conduct researches on sustainable innovation. The results allow establishing deepening layers to analyze the citations and academic connections, including the relationship and connection between the authors' productivity and co-authorship.
\end{abstract}

Keywords: Sustainable Innovation; Eco Innovation; Sustainability; Green Innovation; Bibliometric.

Data do recebimento do artigo: 28/08/2014

Data do aceite de publicação: 15/04/2015

Revista de Administração e Inovação, São Paulo, v. 12, n.3 p. 226-250, jul./set. 2015. 